\title{
Correction to: Down from the treetops: red langur (Presbytis rubicunda) terrestrial behavior
}

\author{
Susan M. Cheyne ${ }^{1,2}$ (D) Supiansyah ${ }^{1} \cdot$ Adul $^{1} \cdot$ Claire J. Neale $^{1} \cdot$ Carolyn Thompson $^{1} \cdot$ Cara H. Wilcox $^{1}$. \\ Yvette C. Ehlers Smith ${ }^{1,3}$. David A. Ehlers Smith ${ }^{1,3}$
}

Published online: 9 August 2018

(C) Japan Monkey Centre and Springer Japan KK, part of Springer Nature 2018

\section{Correction to: Primates \\ https://doi.org/10.1007/s10329-018-0676-5}

In the original publication of this article, the Table 2 was published incorrectly. The revised Table 2 is given on the following page.

The original article can be found online at https://doi.org/10.1007/ s10329-018-0676-5.

Susan M. Cheyne

s.cheyne@borneonature.org

1 Borneo Nature Foundation, J1. Bukit Raya Induk No. 82,

Palangka Raya, Indonesia

2 Faculty of Humanities, Social Sciences and Law, Oxford Brookes University, Oxford, UK

3 University of KwaZulu-Natal, School of Life Sciences,

Private Bag X01, Scottsville, Pietermaritzburg 3209,

South Africa 
Table 2 Number of independent photos of red langurs on the ground across all sites as detected by the cameras

\begin{tabular}{|c|c|c|c|c|c|}
\hline & Habitat & $\begin{array}{l}\text { Total images/ } \\
\text { sightings }\end{array}$ & $\begin{array}{l}\text { Logging status/age } \\
\text { (years) }\end{array}$ & Fire & Hunting \\
\hline Bawan 2010 & Kerangas/ombrogenous peat-swamp forest (PSF) mosaic & 3 & Current & $\mathrm{Y}$ & $\mathrm{Y}$ \\
\hline Bawan 2012 & Kerangas/ombrogenous peat-swamp forest (PSF) mosaic & 8 & Current & $\mathrm{Y}$ & $\mathrm{Y}$ \\
\hline Sabangau $^{\mathrm{a}}$ & Ombrogenous PSF & $3(37)$ & $>10$ & $\mathrm{Y}$ & $\mathrm{Y}$ \\
\hline Murung Raya & Lowland montane & 1 & $>10$ & $\mathrm{~N}$ & $\mathrm{Y}$ \\
\hline Kutai & Lowland dipterocarp & 0 & $>10$ & $\mathrm{~N}$ & Y \\
\hline Sungai Wain & Alluvial peat-swamp forest & 0 & $>20$ & $\mathrm{~N}$ & $\mathrm{~N}$ \\
\hline Mungku Baru & Kerangas/ombrogenous peat-swamp forest (PSF) mosaic & 21 & $>20$ & $\mathrm{Y}$ & $\mathrm{Y}$ \\
\hline Lesan & Lowland dipterocarp & 40 & $>20$ & $\mathrm{~N}$ & $\mathrm{Y}$ \\
\hline Belantikan & Lowland dipterocarp & 1 & $>20$ & $\mathrm{Y}$ & $\mathrm{Y}$ \\
\hline
\end{tabular}

$0=$ not detected. Fire and hunting: $\mathrm{Y}=$ has occurred and/or still occurring, $\mathrm{N}=$ has never occurred. Logging status: current $=$ ongoing, $>10=$ logging occurred more than 10 years ago but has stopped, $>20=$ logging occurred more than 20 years ago but has stopped

${ }^{a}$ Sabangau data are from the 6-month comparison survey period, data in brackets are numbers from the full 7 years of survey (Adul et al. 2015) 